\title{
Memory deficits following lesions of hippocampus or amygdala in rat: Assessment by an object-memory test battery
}

\author{
DAVE G. MUMBY, JOHN P. J. PINEL, TOM J. KORNECOOK, \\ MARTIN J. SHEN, and VAN A. REDILA \\ University of British Columbia, Vancouver, British Columbia, Canada
}

\begin{abstract}
We tested rats with bilateral lesions of the hippocampus or the amygdala on a battery of five object-memory tasks, which resemble those that have been used in the study of amnesia in humans and monkeys: (1) object discrimination, (2) discrimination reversal, (3) eight-pair concurrent object discrimination, (4) nonrecurring-items delayed nonmatching-to-sample (DNMS) with retention delays of $4,15,30,60$, and $120 \mathrm{sec}$ and with lists of three, five, and seven samples, and (5) order discrimination. All testing was postsurgery. Relative to control rats, the rats with hippocampal lesions required more trials to master the object discrimination and the concurrent object discrimination. Rats with hippocampal lesions required about as many trials as did control rats to master DNMS at the 4sec delay and displayed only mild deficits at the longest (120-sec) delay. Rats with amygdalar lesions required more trials to master the concurrent object discriminations than did controls, but significantly fewer than did rats with hippocampal lesions. They required more trials than did controls to master DNMS with a 4-sec delay; however, after they had done so, they continued to perform normally as the delay was increased. Neither lesion produced deficits on discrimination reversal, DNMS with lists, or order discrimination. While these findings demonstrate that the mnemonic effects of hippocampal and amygdalar lesions can be dissociated using a battery of object-memory tasks, they also suggest a limited role for these two structures in many object-memory abilities.
\end{abstract}

Studies of amnesia in monkeys typically involve memory tasks that require the monkeys to remember information about objects; for example, two-choice object discrimination, multiple-pair concurrent object discrimination, and nonrecurring-items delayed matching- or nonmatching-to-sample (DNMS). Similar object-memory tasks have been used to assess the memory deficits of human amnesics with results virtually identical to those in monkeys with similar brain damage (Aggleton, Nicol, Huston, \& Fairbairn, 1988; Aggleton, Shaw, \& Gaffan, 1992; Squire, Zola-Morgan, \& Chen, 1988). These results indicate a close correspondence between human and nonhuman primates in terms of the neuroanatomical bases of various object-memory abilities (see review by Squire, 1992).

The present experiment sought to broaden the comparative basis for the study of object memory to include rats. Historically, differences in the memory tasks that have been used with rodents and primates have obscured the extent to which the mnemonic abilities and the neural

This research was supported by a B.C. Health Care Research Foundation grant to J.P.J.P. D.G.M. was supported by a postgraduate studentship from the Medical Research Council of Canada. The authors wish to thank Robert Sutherland and the two anonymous reviewers for their helpful comments on this paper. Correspondence should be addressed to D. G. Mumby, Department of Psychology, DS-413, Concordia University, 7141 Sherbrooke St. W., Montreal, Quebec, Canada H4B 1R6 (e-mail: mumby@vax2.concordia.ca). bases of these abilities are similar across these two mammalian orders. Most memory tasks for monkeys require them to retain information about objects, whereas most memory tasks for rats require them to retain information about places; thus, because the neural systems that underlie memory for objects and memory for places are anatomically different (Goldman-Rakic, 1988; O'Keefe \& Nadel, 1978; Ungerleider \& Mishkin, 1982), it is difficult to compare the findings from studies of brainmemory relations in monkeys and rats. To facilitate such comparisons, we adapted the following object-memory tasks for use with rats: (1) two-choice object discrimination, (2) discrimination reversal, (3) eight-pair concurrent object discrimination, (4) nonrecurring-items DNMS, and (5) order-discrimination (Mumby, Pinel, \& Anzarut, 1991). Each task requires the acquisition, retention, and expression of either qualitatively or quantitatively different kinds of information, and for this reason, they have been useful in dissociating the mnemonic effects of damage to different brain structures in monkeys (Squire, 1992). However, because object-memory tasks have only recently been developed for rats (e.g., Mumby et al., 1991; Mumby, Pinel, \& Wood, 1990; Rothblat \& Hayes, 1987; Santucci \& Treichler, 1990; Wible, Shiber, \& Olton, 1992), it is not yet clear whether similar dissociations occur in rats.

This experiment had two specific aims. The first aim was to demonstrate that the object-memory abilities of rats can be tested in the same way as those of humans 
and monkeys; the second aim was to compare the mnemonic effects of lesions of the hippocampal formation (cornu ammonius, dentate gyrus, and subicular complex) and the amygdala using our battery of object-memory tasks. The main reason why we chose to examine the effects of hippocampal and amygdalar lesions was that such lesions have different effects on the object-memory abilities of monkeys (Squire, 1992). Another reason was that we wanted to compare the results of this experiment with the results of a previous experiment in which we assessed the effects of hippocampal and amygdalar lesions on DNMS (Mumby, Wood, \& Pinel, 1992). The rats in that earlier study received extensive presurgery DNMS training, whereas the rats in the present experiment did not. Thus, we were able to assess the degree to which extensive pretraining was responsible for the mild DNMS deficits that we observed in rats with hippocampal or amygdalar lesion in our previous experiment. Presurgery DNMS training in monkeys has been shown to reduce the deficits produced by hippocampal lesions (Murray, 1990).

\section{METHOD}

\section{Subjects}

Twenty adult male Long-Evans hooded rats served as subjects. They were divided into four groups of 5 rats each: (1) rats in Group HPC received bilateral aspiration lesions of the dorsal hippocampus and the overlying parietal cortex, and they also received electrolytic lesions of the ventral portions of the hippocampal formation; (2) rats in Group AM received bilateral electrolytic lesions of the amygdala; (3) rats in Group CONT received sham surgery and sustained no brain damage; and (4) rats in Group PC received bilateral aspiration lesions of the parietal cortex, and thus, they served as controls for the cortical damage sustained by the rats in Group HPC.

\section{Surgery}

All surgery was performed under pentobarbitol anesthesia $(60 \mathrm{mg} / \mathrm{kg}$, i.p.). Atropine sulphate $(0.2 \mathrm{ml}, 0.4 \mathrm{mg} / \mathrm{ml}$, i.p.) was administered approximately $20 \mathrm{~min}$ prior to anaesthetization to reduce mucous secretion.

The hippocampal lesions were made using a combination of aspiration to remove the dorsal hippocampus and electrolysis to remove the ventral portion of the hippocampus, dentate gyrus, and subiculum. In preparation for hippocampal surgery, the scalp was incised and holes were cut in the skull over each hemisphere with a dental drill; the holes extended from approximately $2 \mathrm{~mm}$ posterior to the coronal suture to $2 \mathrm{~mm}$ anterior to the lamboid suture and from $1.5 \mathrm{~mm}$ lateral to the sagittal suture to within $1 \mathrm{~mm}$ of the temporal ridge.

The electrolytic lesions of the ventral hippocampal formation were made first, followed immediately by the aspiration lesions of the dorsal hippocampus. The electrolytic lesions were made bilaterally at two sites $(2 \mathrm{~mA}$ for $20 \mathrm{sec})$ with a bipolar stainless steel electrode that was insulated with Teflon except for approximately $1 \mathrm{~mm}$ at its tip. The following were the electrode coordinates, in millimeters, relative to bregma: AP -4.8, ML 4.6, DV -8.0; AP $-5.8, \mathrm{ML} 4.6, \mathrm{DV}-7.7$. For the aspiration lesions, the dura mater was cut and a portion of the underlying parietal cortex and white matter was aspirated with a glass Pasteur pipette, exposing the dorsal hippocampus. The dorsal hippocampus was aspirated, the cavity was filled with Gelfoam (Upjohn Co., Don Mills, Ontario), and the skin was sutured. Diazepam $(10-15 \mathrm{mg} / \mathrm{kg}$, i.p.) was administered as soon as the rat began to regain consciousness; for the next
$24 \mathrm{~h}$, smaller doses were periodically administered to control convulsions that we have sometimes observed in rats following hippocampal lesions. We did not observe seizures in any of the present rats with hippocampal lesions.

The amygdalar lesions were made bilaterally at four sites with an electrode similar to the one that was used to make the electrolytic component of the hippocampal lesions. The following were the electrode coordinates, in millimeters, relative to bregma: $\mathrm{AP}-1.5, \mathrm{ML} 4.4, \mathrm{DV}-9.6(2 \mathrm{~mA}$ for $10 \mathrm{sec})$; $\mathrm{AP}-2.3, \mathrm{ML} 4.5$, DV $-9.8(2 \mathrm{~mA}$ for $20 \mathrm{sec})$; AP -3.3 , ML 4.5, DV $-10.0(2 \mathrm{~mA}$ for $20 \mathrm{sec})$; $\mathrm{AP}-4.3, \mathrm{ML} 4.5, \mathrm{DV}-10.2(2 \mathrm{~mA}$ for $20 \mathrm{sec})$.

The procedures for making the parietal cortex lesions were identical to those for removing this overlying cortex prior to the hippocampal aspirations. The sham-surgery controls received a scalp incision, but no damage was done to either the skull or the brain. The rats were allowed to recover from surgery for 14 days before behavioral training commenced.

Upon completion of behavioral testing, the rats were administered an overdose of sodium pentobarbitol and perfused transcardially with saline, followed by $10 \%$ formol-saline. The brains were removed and stored in $10 \%$ formol-saline. They were frozen and cut on a cryostat in the coronal plane with a thickness of $30 \mu \mathrm{m}$. Every 10th section was mounted on a glass slide and stained with cresyl violet.

\section{Apparatus}

All of the tests were conducted in the same apparatus, which has been described in detail elsewhere (Mumby et al., 1990). Briefly, it consisted of an elevated runway, which was separated from identical goal areas at each end by opaque guillotine doors. Each goal area contained two food wells into which food pellets (45-mg BioServ Inc., Frenchtown, NJ) could be delivered by hand through plastic tubes that were mounted on the outside of the apparatus. The two food wells were separated by a short divider wall, which protruded from the center of the end wall. The sides of the goal areas were open to allow the experimenter to easily place objects over the food wells and to remove them.

The test stimuli for all of the tasks were taken from a pool of over 400 objects of various shapes, sizes, colors, and textures. Each object was large enough to cover a food well but light enough to be easily displaced by the rats. Different objects were used in each of the tasks. The objects were washed every few days in a solution of water and chlorine bleach.

\section{Procedure}

All testing occurred during the light phase of the light:dark cycle, between 14 and $21 \mathrm{~h}$ after a rat's most recent meal. Each rat was tested no more than once per day and no fewer than four times per week; no more than 2 days intervened between consecutive test sessions. The rats were not handled during a session once they had been placed in the apparatus.

Following recovery from surgery, each rat was habituated to the apparatus (see Mumby et al., 1990) and then was trained on each of the five object-memory tasks. For all rats, the tasks were administered in the order in which they are described below.

Task 1: Object discrimination. This task assessed the rats' ability to learn which of two objects was associated with food reward. For all of the rats, the same two objects served as the stimuli on all trials. One of the objects was designated S+ (rewarded); the other object was designated $\mathrm{S}-$ (not rewarded). To begin each session, the rat was placed in the center compartment of the apparatus; one door was open and the other was closed. S+ and S- were placed over the food wells behind the closed door-the position of S+ (left or right) varied from trial to trial according to a pseudorandom pattern. The experimenter opened the door to expose the two objects. As the rat approached and displaced one of the objects, the experimenter lowered the far door in preparation for the 
next trial. If the rat displaced $S+$, a food pellet was delivered to that food well; if the rat displaced $S-$, no food pellet was delivered. The experimenter then positioned $S+$ and $S-$ at the other end of the apparatus to begin the next trial. There were 25 trials per session and an intertrial interval of approximately $15 \mathrm{sec}$. Training for each rat continued until it chose $\mathrm{S}+$ on at least 22 trials (i.e., $88 \%$ correct) on two consecutive sessions.

Task 2: Discrimination reversal. This task assessed the rats' ability to respond appropriately to a change in reinforcement contingency-- to form a new object-reward association that was incompatible with a previous one and to use the new association to guide its behavior. The same two objects were presented to the rats as they had been for the object-discrimination task, but the object that was previously $\mathrm{S}-$ became $\mathrm{S}+$, and vice versa. Training for each rat continued until it chose the new $S+$ on at least 22 trials (i.e., $88 \%$ correct) on two consecutive sessions.

Task 3: Eight-pair concurrent object discrimination. This task assessed the rats' ability to simultaneously learn several different object-reward associations. Sixteen objects were divided into eight pairs. One of the objects in each pair was designated $\mathrm{S}^{+}$, and the other one was designated $\mathrm{S}-$. The two objects in each pair always appeared together, and each pair was presented five times per session in the following order: Pair 1, Pair 2,... Pair 7, Pair 8, Pair 1, Pair 2, and so on. All other procedures were the same as those for the objectdiscrimination task. The intertrial interval was approximately $15 \mathrm{sec}$; thus, the interval between each presentation of a particular pair was approximately $120 \mathrm{sec}$. Training continued for each rat until it chose $\mathrm{S}+$ on at least 36 trials out of 40 (i.e., $90 \%$ correct) on two consecutive sessions. After the rats achieved this criterion with the first eight pairs of objects (Problem Set A), they received identical training with eight different pairs of objects (Problem Set B).

Task 4: Delayed nonmatching-to-sample (DNMS). The procedures for the DNMS task have previously been described in detail (see Mumby et al., 1990). It is modeled after the primate DNMS task that was developed by Gaffan (1974) and elaborated by Mishkin and Delacour (1975). A collection of 350 objects were divided into seven sets of 50 . A different set was used on successive sessions, and different objects were used as stimuli on successive trials within each session. At the beginning of each trial, the rat approached and displaced a sample object from over a baited well at one end of the apparatus. Following a retention delay during which the sample was concealed, it was presented again during a test phase, along with a different (i.e., novel) object. The rat received a food pellet if, during the test phase, it displaced the novel object, but not if it displaced the sample object.

There were three phases of DNMS training and testing: (1) Acquisition of the DNMS task with a brief (i.e., 4-sec) retention delay. The use of such a brief delay between the sample and test phases of a trial places only a small demand on rats' ability to retain information about the identity of the sample object. Therefore, the rate at which a rat masters the DNMS task at this brief delay is a good index of its ability to learn, retain, and apply the nonmatching principle. Training for each rat continued at the 4-sec delay until it chose the novel object on at least 17 trials out of 20 (i.e., $85 \%$ correct) on two consecutive sessions. (2) Testing at delays of $15,30,60$, and $120 \mathrm{sec}$. This phase of DNMS testing assessed the rats' ability to retain information about the identity of an object over various delay intervals. Each rat received six sessions ( 20 trials per session) at each delay before moving onto the next longest delay. (3) Testing with "lists" of three, five, and seven sample objects. This phase of testing assessed the rats' ability to retain various amounts of information over a delay interval. On each trial, the rats were first presented with a sequence of sample objects, one every $15 \mathrm{sec}$, which they were permitted to displace from over baited wells; successive sample objects in the list were presented at alternate ends of the apparatus. Approximately $15 \mathrm{sec}$ after the presentation of the last sample in the list, the first sample in the list was presented again, along with a novel object, and then the remaining samples from the list were presented at 15 -sec intervals, each with a different novel object. As before, the rat was rewarded for selecting the novel objects. Each rat received seven sessions with each list length before moving onto the next longest list. On sessions with lists of three sample objects, eight lists were presented (i.e., 24 trials per session); on sessions with lists of five sample objects, five lists were presented (i.e., 25 trials per session); on sessions with lists of seven sample objects, three lists were presented (i.e., 21 trials per session).

Task 5: Order discrimination. This task assessed the rats' ability to remember the order in which a sequence of objects had been presented to them. This task was similar to one developed for monkeys by Gower (1992), which in turn was modeled after a protocol used to study humans' serial-order memory (Hacker, 1980). The procedure was similar to DNMS with a list of five sample objects, except that $15 \mathrm{sec}$ after the presentation of the last object in the list, two of the objects from the list were presented together. The rat was rewarded if it displaced the object that had been presented earlier in the sample list. The difference in the two objects' position in the list (referred to as the "lag") could be either three (Sample 1 vs. Sample 4; Sample 2 vs. Sample 5), two (Sample 1 vs. Sample 3; Sample 2 vs. Sample 4; Sample 3 vs. Sample 5), or one (Sample 1 vs. Sample 2; Sample 2 vs. Sample 3; Sample 3 vs. Sample 4; Sample 4 vs. Sample 5), and each of the possible pairings were counterbalanced within each lag condition. In humans and monkeys, order-discrimination ability decreases as the separation lag between comparison stimuli decreases (Gower, 1992; Hacker, 1980). We sought to determine whether rats encode order information about lists of objects and whether there are parametric parallels among rats and primates in terms of their order-memory abilities. The smaller the lag, the more difficult it should be to discriminate which of the two objects came earlier in the list. Each rat received seven sessions with a lag of three, then seven sessions with a lag of two, then seven sessions with a lag of one. There were 10 trials per session (i.e., 10 lists of five samples, with a test following the presentation of each list).

\section{Statistical Analyses}

The primary performance measure for the object discrimination, discrimination reversal, concurrent object discriminations, and the acquisition stage of DNMS testing was the number of trials that each rat required to reach the respective performance criteria. The trials of the criterion sessions were not included in the calculation of this measure. Following analysis of these data with analysis of variance (ANOVA), we performed planned comparisons with all possible group pairings, using variance estimates based only on the two groups involved in each comparison instead of a pooled variance estimate. The number of errors-to-criterion was also assessed and subjected to the same analyses.

The primary performance measure on DNMS at the various retention delays and list lengths, and on the order-discrimination task, was percent correct. These data were analyzed with repeated measures ANOVAs using group (lesion type) as a between-subjects factor and session (sessions within each condition) and condition (i.e., retention delay, list length, or lag) as within-subjects factors. We also conducted planned pairwise comparisons among the groups in each condition of each task, using variance estimates based only on the two groups involved in each comparison.

\section{RESULTS}

\section{Histological}

Figure 1 illustrates the location and the extent of the lesions. The hippocampal lesions included the entire dorsal hippocampus, most of the lateral and ventral 


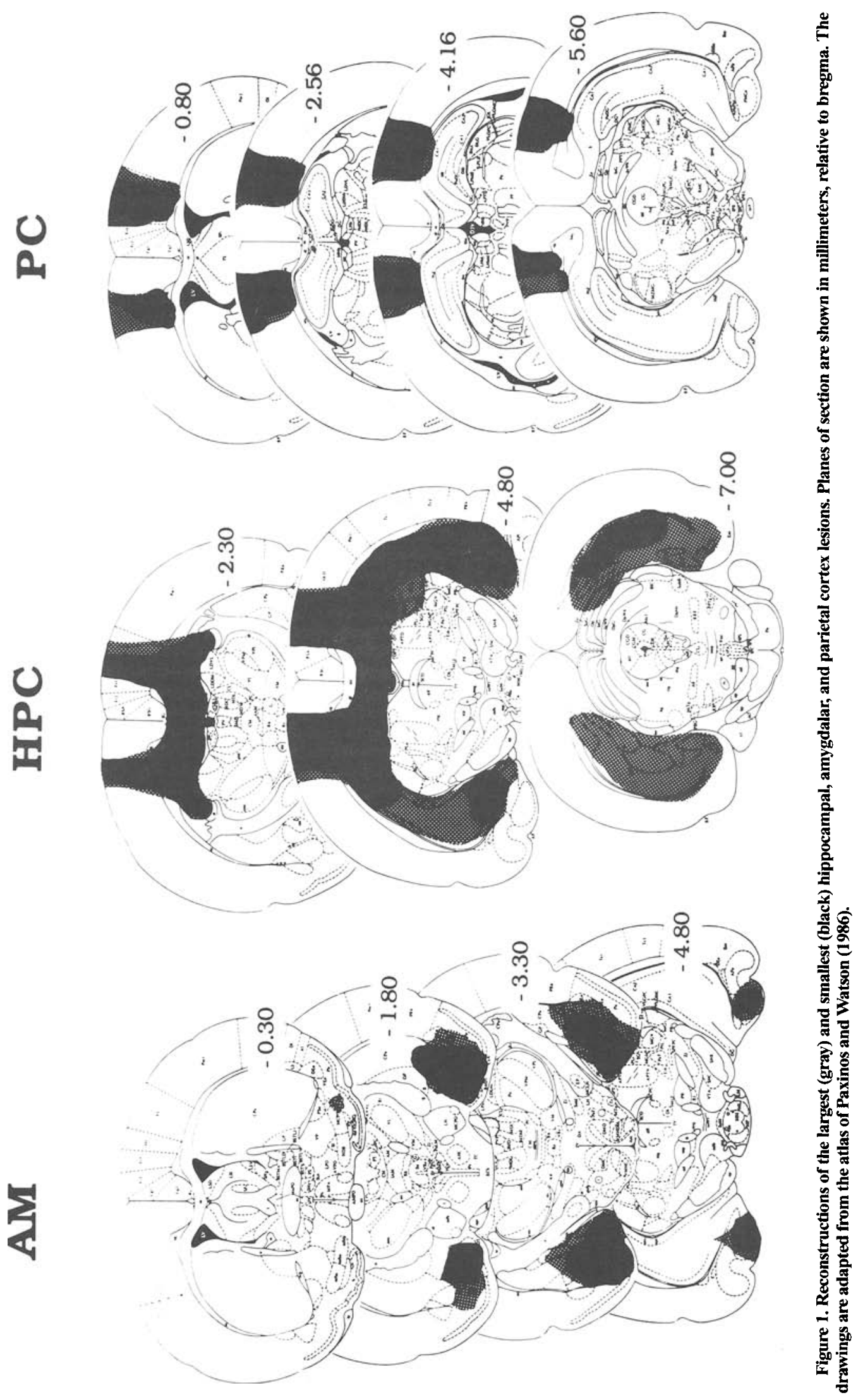


hippocampus, and a portion of the parietal cortex and corpus callosum overlying the dorsal hippocampus. The caudal extent of the hippocampal lesions varied in the amount of presubiculum and parasubiculum that was removed. Each lesion extended rostrally to include the fimbria fornix. Small infarcts were also present in the dorsal thalamus of each of the brains with hippocampal lesions. Most of these were unilateral and involved the habenular nuclei, lateral dorsal nucleus, lateral posterior nucleus, lateral geniculate, and medial geniculate nucleus (see largest lesions in Figure 1). Two of the brains with hippocampal lesions also sustained partial unilateral damage to the colliculi.

As intended, the area of parietal cortex that was removed in the rats of Group PC tended to be slightly larger than that in the rats of Group HPC. The same area of cortex that tended to be removed in the rats of Group HPC were also removed in every one of the parietal cortex lesions. The area of cortex that was removed in both groups included posterior portions of areas FL and Fr1, virtually all of area HL, superior portions of Par1, and anterior portions of Oc2MM, Oc2ML, and Oc2L (Zilles, 1985). There tended to be slightly more damage to white matter in the rats with HPC lesions than in the rats with PC lesions. Two of the rats with PC lesions sustained bilateral damage to the alveus, and in both of these rats a small portion of the dorsal CA1 region was damaged unilaterally (see the largest PC lesion in Figure 1); one rat with PC lesions sustained unilateral damage to the alveus.

The amygdalar lesions varied in the extent of damage to specific nuclei, but there was consistent damage to the medial two thirds of the amygdaloid complex. No specific amygdaloid nuclei were consistently spared. The caudal extent of the amygdalar lesions in 2 rats included small portions of medial entorhinal cortex in both hemispheres (see the largest AM lesion in Figure 1); in 1 other AM-lesioned rat, the medial entorhinal cortex sustained slight unilateral damage. The lesions of one of the rats in this group extended much more posterior than in any of the others; it included portions of the ventral subiculum and dentate gyrus bilaterally. This rat also had frequent seizures, which sometimes occurred just before or after a test session. Because none of the other rats in this experiment were ever seen to display seizures, the data from this rat were omitted from all analyses.

\section{Behavioral}

Errors-to-criterion proved to be a less sensitive measure than did trials-to-criterion, and it revealed no significant effects of the lesions beyond those revealed by the latter measure. Therefore, only the trials-to-criterion data are described and presented graphically for the object discrimination, discrimination-reversal, and concurrentobject-discrimination tasks, and for the acquisition phase of the DNMS task.
Task 1: Object discrimination. Figure 2 (top) illustrates the mean number of trials that the rats in each group required to reach the criterion of at least $88 \%$ correct on two consecutive object-discrimination sessions. An ANOVA revealed a significant group effect $[F(3,15)=$ $3.26, p=.05]$. Planned comparisons revealed that the rats in Group HPC required significantly more trials than did the rats in Groups PC $[t(8)=3.04, p=.016]$ and CONT $[t(8)=2.72, p=.026]$. None of the other differences among the groups was statistically significant (all $p \mathrm{~s}>.20$ ).

Task 2: Discrimination reversal. Figure 2 (bottom) illustrates the mean number of trials that the rats in each group required to reach the criterion of at least $88 \%$ correct on two consecutive discrimination-reversal sessions. An ANOVA revealed a nonsignificant group effect $[F(3,15)=3.05, p=.061]$. The rats in Group HPC required many more trials than did those in the other three groups; however, none of the pairwise differences among the groups was statistically significant (all $p s>.05$ ).

Task 3: Eight-pair concurrent object discrimination. Figure 3 illustrates the mean number of trials that the rats in each group required to reach the criterion of at least $90 \%$ correct on two consecutive concurrent-objectdiscrimination sessions. When the data from both Problem Set A and Problem Set B were combined for each rat, an ANOVA revealed a significant group effect $[F(3,15)$ $=19.25, p<.001]$. The rats in Groups HPC and AM required more trials to reach the criterion than did the rats
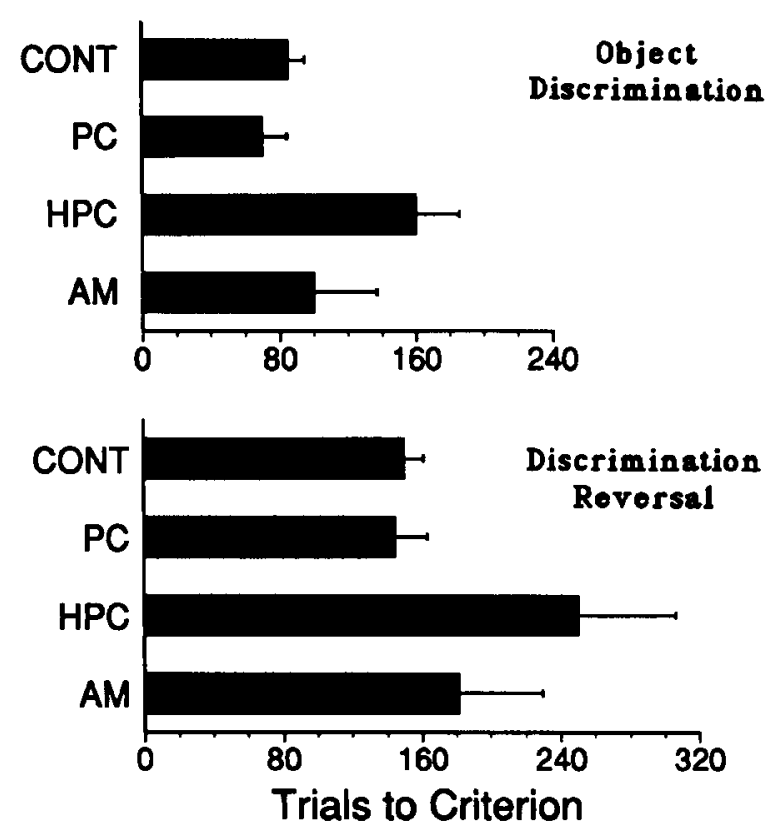

Figure 2. Mean number of trials that the rats in each group required to reach the criterion on the object-discrimination task (top) and the discrimination-reversal task (bottom). Error bars represent SEMS. 


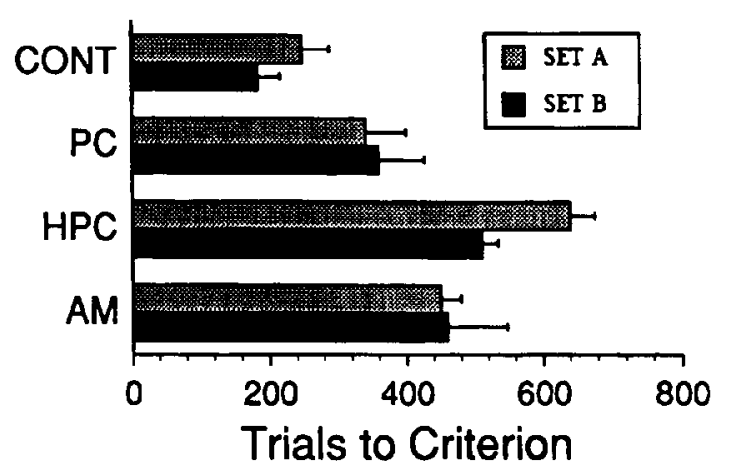

Figure 3. Mean number of trials that the rats in each group required to reach the criterion on Problem Set $A$ and Problem Set $B$ of the concurrent-object-discrimination task. Error bars represent SEMS.

in Group CONT [HPC vs. CONT, $t(8)=11.20, p<$ $.0001 ; \mathrm{AM}$ vs. CONT $t(7)=4.27, p<.005]$, and the rats in Group HPC required more trials than did the rats in Group PC $[t(8)=4.25, p<.005]$ and Group AM $[t(7)=$ $3.23, p=.014]$. In sum, hippocampal and amygdalar lesions produced deficits in concurrent object discrimination, but the deficit was greater following hippocampal lesions.

Task 4: Delayed nonmatching-to-sample (DNMS). Figure 4 illustrates DNMS acquisition and DNMS per- formance across the different delay and list-length conditions. An ANOVA revealed a significant group effect on trials-to-criterion during acquisition $[F(3,15)=3.79$, $p=.032]$. The rats in Group AM required significantly more trials to reach the criterion of at least 17 correct trials out of 20 on two consecutive sessions than did the rats in Group HPC $[t(7)=3.13, p=.016]$; they also required more trials than did the rats in Group CONT $[t(7)=2.22, p=.060]$ and Group PC $[t(7)=2.03, p=$ .079 ], but these differences were not statistically significant. None of the other differences among the groups was statistically significant (all $p s>.10$ ).

Despite being impaired in acquisition of DNMS at the 4-sec delay, the rats in Group AM later performed normally at delays of up to $120 \mathrm{sec}$. In contrast, the rats in Group HPC displayed normal acquisition, but they were subsequently impaired, relative to each of the other three groups, at the 120-sec delay (CONT, $p=.002 ; \mathrm{PC}, p=$ .033 ; AM, $p=.050$ ). The rats in Group PC were impaired relative to those in Group CONT at the 120 -sec delay $(p=.045)$.

Scores decreased as the sample list length increased $[F(2,30)=18.37, p<.0001]$. However, there were no significant differences among the groups during this stage of DNMS testing.

Task 5: Order discrimination. Figure 5 shows that scores on the order-discrimination task decreased as the lag decreased $[F(2,30)=24.47, p<.0001]$. However,
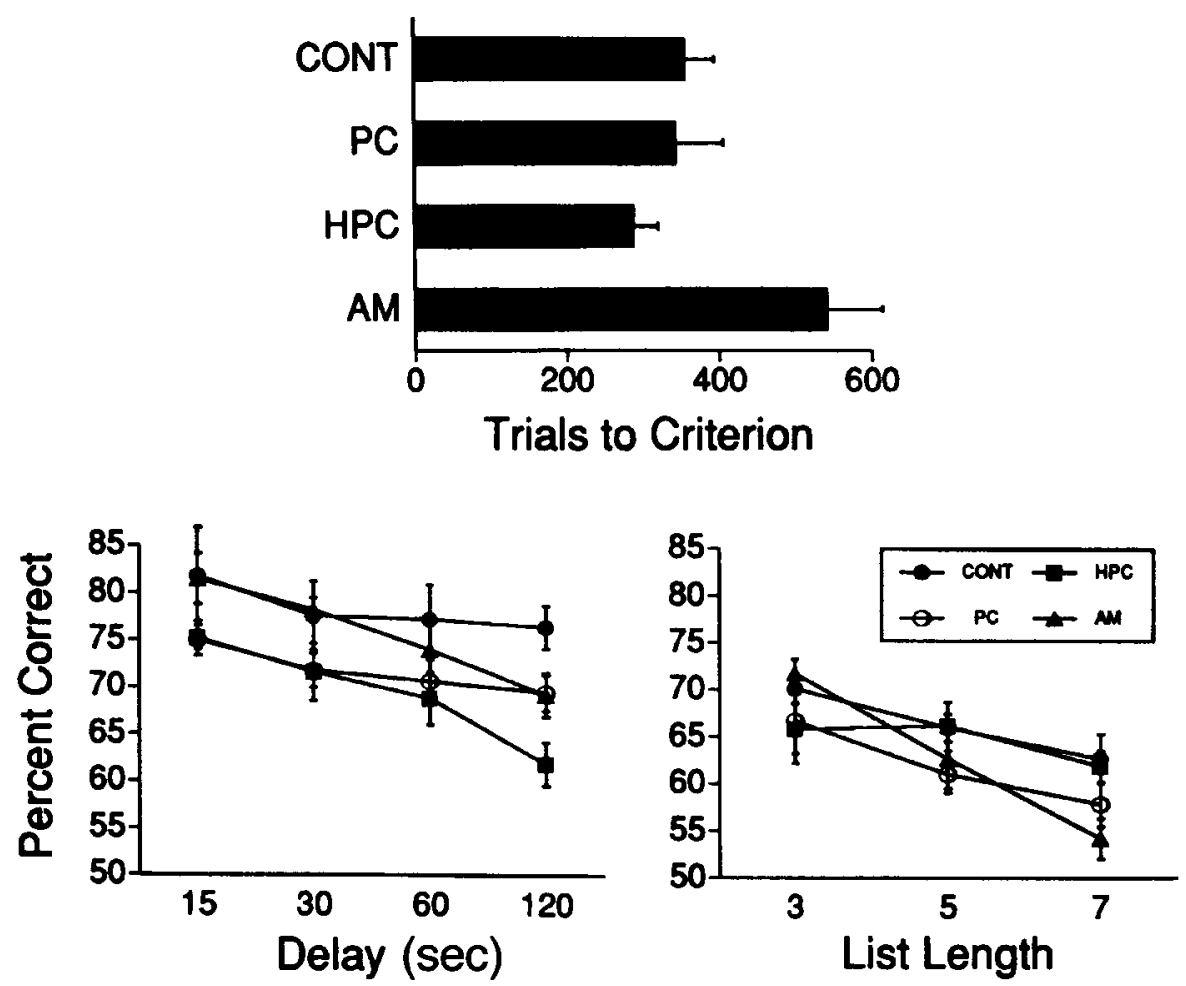

Figure 4. Mean number of trials that the rats in each group required to reach the criterion on the DNMS task at the 4-sec delay. Mean percent correct in each group on DNMS at different retention delays and sample-list lengths. Error bars represent $S E M$ s. 


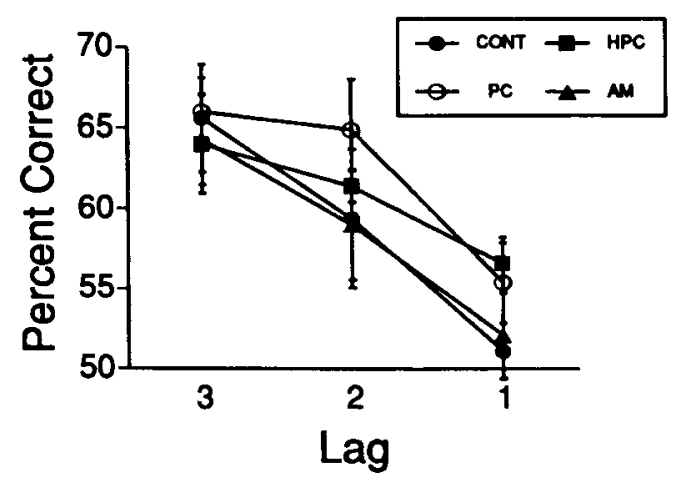

Figure 5. Mean percent correct in each group on the orderdiscrimination task. Error bars represent SEMs.

there were no significant differences among the groups on this task. Furthermore, none of the groups displayed serial-position effects within any of the lag conditions. For example, within-group performance was not significantly different on the lag-of-three trials on which Samples 1 and 4 were paired and on those trials on which Samples 2 and 5 were paired (all $p s>.10$ ); within-group performance was also similar on all lag-of-two trial types (all $p \mathrm{~s}>.08$ ) and on all lag-of-one trial types (all $p \mathrm{~s}>.15$ ).

\section{DISCUSSION}

The object-memory tasks that were used in the present experiment resemble in key respects some of the object-memory tasks that have been employed in studies of amnesia in monkeys, and each of them required the rats to acquire, retain, and express information that differs from that of the other tasks in the battery. Rats with bilateral lesions of the hippocampus or the amygdala displayed different patterns of deficits across these tasks. It should be emphasized that most of the observed deficits were in the rate at which lesioned rats mastered certain tasks. With the exception of the mild DNMS deficits observed in rats with hippocampal lesions at the 120 -sec delay, rats with hippocampal or amygdalar lesions were eventually able to reach the same performance levels as the controls on every task; it is possible that, with additional testing at the 120 -sec delay, the mild DNMS deficits in rats with hippocampal lesions would also have disappeared. One interpretation of acquisition deficits such as those that we observed is that they reflect the loss of some function that is not essential for normal task performance but that normally plays a role in the establishment of effective behavioral or cognitive strategies for solving the task-that is, it takes longer for the lesioned rats to establish either the strategies that normal rats use to solve the task or equally effective alternative strategies for solving it. Put another way, some of the processes that mediate performance while a task is being learned are different from those that maintain performance after the task is solved. The point is that the functions of the hippocampus, the amygdala, and the parietal cortex are not necessary for normal performance of any of the object-memory tasks in this experiment.

Each of the present tasks uses the same apparatus and similar stimuli (i.e., objects), and performance on each task is motivated by food and requires the same operant response (i.e., displacement of an object). These similarities make it unlikely that the observed dissociations were due to different motivational, perceptual, or motor effects of hippocampal or amygdalar lesions. The fact that certain task variables affect normal rats' and monkeys' performance on these tasks in qualitatively similar ways provides strong support for the view that these tasks assess similar mnemonic processes in the two species. For example, increasing either the retention delay or the number of objects in a sample list decreases the accuracy of DNMS performance in rats and monkeys. Accordingly, comparisons of the DNMS performance of rats and monkeys with similar lesions should provide insights into the neural circuitry underlying object recognition in these two species.

The object-discrimination task requires rats to learn which of two objects is associated with food reward. Our rats with hippocampal lesions needed significantly more trials to master this problem than did control rats or rats with amygdalar lesions. This finding is consistent with reports of deficits in the rate at which monkeys with hippocampal lesions acquire an object discrimination (ZolaMorgan, Squire, \& Amaral, 1989b), but it is inconsistent with reports of normal object-discrimination learning in rats with hippocampal lesions (Wible et al., 1992). One potentially important difference between the present experiment and that of Wible et al. was that their rats received presurgery object-discrimination training, whereas our rats did not. We recently found that pretrained rats with ischemia-induced hippocampal damage learned an object-discrimination problem at a normal rate (Wood, Mumby, Pinel, \& Phillips, 1993). It should be noted that nonpretrained monkeys with hippocampal lesions learn some visual discriminations (e.g., pattern discriminations) at a normal rate (e.g., Zola-Morgan et al., 1989b), and nonpretrained rats with hippocampal lesions can also learn some visual discriminations (e.g., brightness discriminations) at a normal rate (Kimble, 1963). The present findings demonstrate that hippocampal-lesion effects can be obtained on a task that requires neither working (Olton, Becker, \& Handelmann, 1979) nor spatial (O'Keefe \& Nadel, 1978) memory. The lack of a deficit on the object-discrimination task in our rats with amygdalar lesions is consistent with similar reports in rats (e.g., Kentridge, Shaw, \& Aggleton, 1991) and monkeys (Horel, Keating, \& Misantone, 1975).

The discrimination-reversal task requires rats to respond appropriately to a change in the reinforcement contingency; the rats must form a new object-reward association that is incompatible with an existing one and use it to guide their behavior. None of the lesions produced statistically significant deficits on this task. Our findings are thus consistent with other reports that rats 
with amygdalar lesions (Kentridge et al., 1991) and monkeys with hippocampal lesions (Jones \& Mishkin, 1972) learn a single reversal of an object discrimination at a normal rate. However, our results are inconsistent with reports of impaired discrimination-reversal learning in rats with hippocampal damage (e.g., Kimble, 1963). This discrepancy is probably due to unique features of the present paradigm, such as the use of objects as stimuli or the required operant response of object displacement, as opposed to the more common operant requirement of navigation to a goal location. Consistent with this account, Mahut (1971) observed impaired spatial-reversal learning but normal object-reversal learning in monkeys with hippocampal ablations. The findings on object-discrimination reversal in rats and monkeys appear to be at odds with theories that suggest that the hippocampus is critical for the "flexible" use of nonhippocampal representations in visual memory (e.g., Eichenbaum, Otto, \& Cohen, 1994).

The concurrent-object-discrimination task requires the rats to form concurrently several object-reward associations. It differs from the two-choice discrimination task by introducing interference among the many associations that must be concurrently formed. Rats with either hippocampal lesions or amygdalar lesions displayed deficits on this task, but the deficits were significantly more severe in rats with hippocampal lesions. These findings are consistent with reports of deficits in eightpair concurrent object discrimination in monkeys (Mahut, Zola-Morgan, \& Moss, 1982; Zola-Morgan, Squire, \& Amaral, 1989a) or rats (Wible et al., 1992) with hippocampal lesions. Olton and Shapiro (1993) suggested that hippocampal lesions produce deficits in concurrent object discrimination because hippocampal function is especially critical when stimuli are presented in a way that maximizes interference. However, their hypothesis also predicts that hippocampal lesions should have little effect on a two-pair object discrimination and a substantial effect on discrimination reversals; in fact, we obtained the opposite pattern of results on those two tasks. Still, a high degree of interference on the concurrent-objectdiscrimination task might account in part for the deficits of hippocampal rats.

Our observation of a statistically significant impairment on the concurrent-object-discrimination task in rats with amygdalar lesions is inconsistent with reports in monkeys (e.g., Zola-Morgan et al., 1989a). One possible explanation for this discrepancy is that the damage to medial entorhinal cortex that some of our rats with amygdalar lesions sustained contributed to their deficits; the amygdalar lesions in the Zola-Morgan et al. study did not include the entorhinal cortex. Wible et al. (1992) concluded that the amygdala is unimportant for the acquisition of an eight-pair concurrent object discrimination because rats with lesions of both the hippocampus and the amygdala performed no worse than did rats with lesions of only the hippocampus. The acquisition deficits displayed by our rats with discrete amyg- dalar lesions suggest that Wible et al.'s conclusions were premature.

To master the DNMS task at the 4-sec delay, rats must learn the nonmatching rule and consistently apply it. Our rats with amygdalar lesions required significantly more trials to accomplish this than did control rats or rats with hippocampal lesions. This finding is consistent with reports of normal acquisition by rats with hippocampal lesions of a nonspatial DNMS task in a Y-maze (Aggleton, Hunt, \& Rawlins, 1986) and an object DNMS task (Rothblat \& Kromer, 1991), but they are inconsistent with reports of normal acquisition of the Y-maze DNMS task in rats with amygdalar lesions (Aggleton, Blindt, \& Rawlins, 1989). The present findings are also inconsistent with reports of impaired acquisition of object DNMS in monkeys with hippocampal lesions (ZolaMorgan \& Squire, 1985) and normal acquisition in monkeys with amygdalar lesions (Zola-Morgan et al., 1989a). Monkeys that receive presurgery DNMS training display only mild DNMS deficits following hippocampal lesions, but DNMS performance in monkeys is more susceptible to disruption by hippocampal lesions if the monkeys do not receive any presurgery DNMS training (Murray, 1990). A lack of presurgery DNMS training did not make our rats with hippocampal lesions susceptible to a DNMS acquisition deficit.

The introduction of progressively longer retention delays to the DNMS task requires rats to retain information about the identity of an object for longer periods of time. Our observation of normal DNMS performance over a wide range of retention delays in rats with amygdalar lesions is consistent with reports in monkeys (e.g., ZolaMorgan et al., 1989a) and rats (Mumby et al., 1992). In previous studies, rats with hippocampal lesions also performed normally on object DNMS (Kesner, Bolland, \& Dakis, 1993; Rothblat \& Kromer, 1991) and on a continuous object-recognition task (Jackson-Smith, Kesner, \& Chiba, 1993). Our rats with hippocampal lesions displayed a mild deficit; they were significantly impaired only at the longest (i.e., 120-sec) delay. However, it is possible that neocortical damage may have contributed to these mild DNMS deficits, because rats with parietal cortex lesions also displayed a DNMS deficit at the 120sec delay that was not significantly different from that of the rats with hippocampal lesions. In any event, the DNMS deficits that were displayed by our rats with hippocampal lesions were relatively mild, which indicates that the hippocampus plays, at most, only a minor role in normal object recognition at delays of up to $120 \mathrm{sec}$. We have previously reported mild but statistically significant DNMS deficits at 10-min delays following hippocampal lesions in pretrained rats (Mumby et al., 1992).

The introduction of progressively longer lists of sample objects to the DNMS task requires rats to retain information about the identity of progressively more objects over a delay interval. There were no significant differences among the groups on this task, which sug- 
gests that this ability does not depend upon the functions of the hippocampal formation or the amygdala (or the parietal cortex). The normal performance of our rats with hippocampal lesions on DNMS with lists as long as seven sample objects makes one wonder whether their mild deficit with a single sample object over a 120 -sec delay is a reliable effect of hippocampal lesions. When the list length is seven objects, the delay interval between the sample presentation of any object in the list and the later presentation of the same object in the test phase is approximately $105 \mathrm{sec}$. Although it seems unlikely, one explanation for the present findings is that rats with hippocampal lesions are normal in terms of their ability to remember various amounts of information over delays of up to $105 \mathrm{sec}$, but at some delay between 105 and $120 \mathrm{sec}$ this ability begins to fail, regardless of the amount of information.

When the present DNMS findings are considered together with our previous findings in rats with extensive presurgery DNMS training (Mumby et al., 1992), with the findings of other studies of object DNMS in rats (e.g., Rothblat \& Kromer, 1991), and with the preponderance of recent findings in monkeys (e.g., O'Boyle, Murray, \& Mishkin, 1993), it does not appear that the hippocampus or the amygdala are critical components of the circuitry that underlies normal object-recognition abilities.

The order-discrimination task requires rats to remember information about the order in which a sequence of objects was presented. There were no significant differences among the groups on this task, which suggests that this ability does not depend upon the functions of the hippocampal formation, the amygdala, or the parietal cortex. However, it is possible that the poor performance by control rats on this task, relative to the other tasks in the battery, might have obscured subtle effects of the lesions. Gower (1992) recently demonstrated that monkeys are capable of good performance on an object-based order-discrimination task that is similar to the present one, and it is likely that our rats' performance would have improved with additional training. In retrospect, performance on this task might have been more sensitive to lesion effects if rats were first trained to a high level of competence in the easiest condition (i.e., lag of three) and then given mixed-lag sessions during which the various lags were presented randomly across trials.

Unlike the other tasks in our battery, the orderrecognition task has not yet been used in lesion experiments with monkeys; thus, we cannot compare our present findings with findings in monkeys with hippocampal or amygdalar lesions. Still, the inclusion of this task in the present experiment makes several important contributions. First, our object-based order-discrimination task is similar to a task in which rats are required to remember the order in which a sequence of familiar spatial locations in a radial-arm maze was visited (Kesner \& Holbrook, 1987). The fact that our rats were able to perform the order-discrimination task at above-chance levels with object stimuli that varied across trials indicates that rats encode and store information about the order of objects, as well as the identity of objects. Second, our experiment was the first to assess this ability in rats and, thus, serves to introduce a novel test protocol. Third, the finding of a severely deleterious effect on performance of decreasing the lag between the two objects involved in the order discrimination (see Figure 5) demonstrates the sensitivity of rats' order-discrimination abilities to this parameter. This sensitivity should make the task particularly useful in future experiments on order memory in rats. For example, rats' order memory for spatial locations is impaired by lesions of medial prefrontal cortex (Kesner \& Holbrook, 1987) but not parietal cortex (Kesner \& Gray, 1989), and it would be interesting to know whether a similar dissociation exists for rats' order memory for objects.

Overall, the profiles of object-memory deficits in our rats with hippocampal or amygdalar lesions resemble the profiles that have been reported in monkeys with similar lesions. However, there was one major difference: Rats with amygdalar lesions were impaired in acquisition of DNMS, whereas rats with hippocampal lesions were not; the opposite pattern of results has been reported in some studies with monkeys (e.g., Zola-Morgan et al., 1989a). The following are four possible explanations for this discrepancy: One possibility is that rats and monkeys solve their respective DNMS tasks differently. It is always possible that despite a number of key conceptual and methodological similarities between the rat and monkey DNMS tasks, the two species actually employ different cognitive strategies to solve their respective versions of the task. A second possibility is that the mnemonic functions of the hippocampal formation and the amygdala are different in rats and monkeys; however, there is no compelling evidence for this view (Squire, 1992). A third possibility relates to differences in training histories prior to DNMS training. Our rats were trained on object discrimination, discrimination reversal, and eight-pair concurrent object discrimination, prior to training on the DNMS task. In most experiments with monkeys, DNMS training has preceded training on other tasks. Ideally, the order in which subjects are trained on the various tasks in a battery should be counterbalanced, but this could require a prohibitively large number of subjects in an experiment that also included different lesion groups. A fourth possibility is that there are important differences in the brain damage sustained by the monkeys and rats with ostensibly similar lesions - for example, the presence of rhinal cortex damage in monkeys but not in rats (Mumby \& Pinel, 1994; O'Boyle et al., 1993).

Regardless of the interpretation of any of the individual results obtained in this experiment, the main points to be taken from it are that (1) memory for objects can be tested in the same way in rats as it is in human and nonhuman primates, (2) the mnemonic effects of damage to the hippocampus and the amygdala in rats can be dissociated with a battery of object-memory tasks, (3) the profiles of object-memory deficits in rats with hippocampal and amygdalar lesions are similar in several re- 
spects to those of monkeys with lesions that involve these structures, and (4) the functions of the hippocampus, the amygdala, and the parietal cortex are not essential for normal performance on the present battery of object-memory tasks. Rats with hippocampal, amygdalar, or posterior parietal cortical lesions can (1) learn which of two objects is associated with food reward, (2) form an incompatible object-reward association and respond on the basis of it, (3) form several object-reward associations concurrently, (4) acquire, retain, and consistently apply the nonmatching principle, (5) retain information about the identity of an object over retention delays of up to $120 \mathrm{sec},(6)$ retain information concurrently about the identity of up to seven objects, and (7) encode and retain information about the order in which a sequence of objects was presented. Whereas hippocampal lesions produce permanent impairments of place learning in rats (Barnes, 1988) and amygdalar lesions appear to produce permanent deficits on tasks requiring the association of stimuli with strong affective consequences (e.g., Kesner, 1992), the present findings indicate, at most, a transient and minor role for these two structures in encoding, retaining, and expressing information about the identity and temporal order of objects and their associations with food reward.

The present experiment was the first to use a battery of object-memory tasks to dissociate the mnemonic consequences of damage to the hippocampus and the amygdala in the rat. The similarities that the present tasks bear to the object-memory tasks that are used in studies of amnesia in humans and monkeys facilitate direct comparisons among findings in rats, monkeys, and humans. In addition to providing a highly accessible animal model, the opportunity to study the effects of brain lesions on object memory in rats provides at least one other major advantage over the use of monkeys: stereotaxic surgical procedures enable complete and selective lesions of subcortical structures in rats. In contrast, similar stereotaxic lesions have only recently been made in studies of object memory in monkeys (e.g., O'Boyle et al., 1993; Zola-Morgan et al., 1989a); thus, most previous experiments in monkeys have involved hippocampal and amygdalar lesions that included adjacent cortical structures. This has made it difficult to determine with certainty the contributions that the hippocampus and amygdala make to various object-memory abilities. The development of objectmemory tasks for rats and stereotaxic surgical procedures for monkeys (Alvarez-Royo, Clower, Zola-Morgan, \& Squire, 1991) will help to clarify these issues by making it possible to conduct parallel experiments in these two species.

\section{REFERENCES}

Aggleton, J. P., Blindt, H. S., \& Rawlins, J. N. P. (1989). Effects of amygdaloid and amygdaloid-hippocampal lesions on object recognition and spatial working memory in rats. Behavioral Neuroscience, 103, 962-974.
Aggleton, J. P., Hunt, P. R., \& Rawlins, J. N. P. (1986). The effects of hippocampal lesions upon spatial and non-spatial tests of working memory. Behavioural Brain Research, 19, 133-146.

Aggleton, J. P., Nicol, R. M., Huston, A. E., \& Fairbairn, A. F. (1988). The performance of amnesic subjects on tests of experimental amnesia in animals: Delayed matching-to-sample and concurrent learning. Neuropsychologia, 26, 265-272.

Aggleton, J. P., Shaw, C., \& Gaffan, E. A. (1992). The performance of postencephalitic amnesic subjects on two behavioral tests of memory: Concurrent discrimination learning and delayed nonmatching to sample. Cortex, 28, 359-372.

Alvarez-Royo, P., Clower, R. P., Zola-Morgan, S., \& Squire, L. R. (1991). Stereotaxic lesions of the hippocampus in monkeys: Determination of surgical coordinates and analysis of lesions using magnetic resonance imaging. Journal of Neuroscience Methods, 38, 223-232.

BARNES, C. A. (1988). Spatial learning and memory processes: The search for their neurobiological mechanisms in the rat. Trends in Neurosciences, 11, 163-169.

Eichenbaum, E., Otto, T., \& Cohen, N. J. (1994). Two functional components of the hippocampal memory system. Behavioral \& Brain Sciences, 17, 449-518.

GAFFAN, D. (1974). Recognition impaired and association intact in the memory of monkeys after transection of the fornix. Journal of Comparative \& Physiological Psychology, 86, 1100-1109.

Goldman-Rakic, P. (1988). Topography of cognition: Parallel distributed networks in primate association cortex. Annual Review of Neuroscience, 11, 137-156.

GowER, E. C. (1992). Short-term memory for the temporal order of events in monkeys. Behavioural Brain Research, 52, 99-103.

HACKER, M. J. (1980). Speed and accuracy of recency judgements for events in short-term memory. Journal of Experimental Psychology: Human Learning \& Memory, 6, 651-675.

Horel, J. A., Keating, E. G., \& Misantone, L. J. (1975). Partial Kluver-Bucy syndrome produced by destroying temporal neocortex or amygdala. Brain Research, 94, 347-359.

JACKSON-SMITH, P., KeSNER, R. P., \& CHIBA, A. A. (1993). Continuous recognition of spatial and nonspatial stimuli in hippocampal-lesioned rats. Behavioral \& Neural Biology, 59, 107-119.

JONES, B., \& MishKIN, M. (1972). Limbic lesions and the problem of stimulus-reinforcement associations. Experimental Neurology, 36, 362-377.

Kentridge, R. W., Shaw, C., \& Aggleton, J. P. (1991). Amygdaloid lesions and stimulus-reward associations in the rat. Behavioural Brain Research, 42, 57-66.

KESNER, R. P. (1992). Learning and memory in rats with an emphasis on the role of the amygdala. In J. P. Aggleton (Ed.), The amygdala: Neurobiological aspects of emotion, memory, and mental dysfunction (pp. 379-399). New York: Wiley.

Kesner, R. P., Bolland, B. L., \& Dakis, M. (1993). Memory for spatial locations, motor responses, and objects: Triple dissociation among the hippocampus, caudate nucleus, and extrastriate visual cortex. Experimental Brain Research, 93, 462-470.

KESNER, R. P., \& GRAY, M. L. (1989). Dissociation of item and order memory following parietal cortex lesions in the rat. Behavioral Neuroscience, 103, 907-910.

KeSNER, R. P., \& HolbrooK, R. (1987). Dissociation of item and order spatial memory in rats following medial prefrontal cortex lesions. Neuropsychologia, 25, 653-664.

KimBLE, D. P. (1963). The effects of bilateral hippocampal lesions in rats. Journal of Comparative \& Physiological Psychology, 56, 273-283.

Mahut, H. (1971). Spatial and object reversal learning in monkeys with partial temporal lobe ablations. Neuropsychologia, 9, 409-424.

Mahut, H., Zola-Morgan, S., \& Moss, M. (1982). Hippocampal resections impair associative learning and recognition memory in the monkey. Journal of Neuroscience, 2, 1214-1229.

MishKIN, M., \& Delacour, J. (1975). An analysis of short-term visual memory in the monkey. Journal of Experimental Psychology: Animal Behavior Processes, 1, 326-334.

Mumby, D. G., \& PineL, J. P. J. (1994). Rhinal cortex lesions impair object recognition in rats. Behavioral Neuroscience, 108, 11-18. 
Mumby, D. G., Pinel, J. P. J., \& AnZarut, D. S. (1991). A test battery for assessing nonspatial memory in brain-damaged rats. Society for Neuroscience Abstracts, 17, 130.

Mumby, D. G., Pinel, J. P. J., \& Wood, E. R. (1990). Nonrecurringitems delayed nonmatching-to-sample in rats: A new paradigm for testing nonspatial working memory. Psychobiology, 18, 321-326.

Mumby, D. G., Wood, E. R., \& Pinel, J. P. J. (1992). Object recognition memory in rats is only mildly impaired by lesions of the hippocampus and amygdala. Psychobiology, 20, 18-27.

MurRay, E. A. (1990). Representational memory in nonhuman primates. In R. P. Kesner \& D. S. Olton (Eds.), Neurobiology of comparative cognition (pp. 127-155). Hillsdale, NJ: Erlbaum.

O'Boyle, V. J., Murray, E. A., \& Mishkin, M. (1993). Effects of excitotoxic amygdalo-hippocampal lesions on visual recognition in rhesus monkeys. Society for Neuroscience Abstracts, 19, 438.

O'KeEfE, J., \& NADEL, L. (1978). The hippocampus as a cognitive map. London: Oxford University Press.

Olton, D. S., Becker, J. T., \& Handelmann, G. E. (1979). Hippocampus, space, and memory. Behavioral \& Brain Sciences, 2, 313 365.

Olton, D. S., \& Shapiro, M. L. (1993). Mnemonic dissociations: The power of parameters. Journal of Cognitive Neuroscience, 4, 200-207.

Paxinos, G., \& WATSON, C. (1986). The rat brain in stereotaxic coordinates. Toronto: Academic Press.

Rothblat, L. A., \& Hayes, L. L. (1987). Short-term object recognition memory in the rat: Nonmatching with trial-unique stimuli. $B e$ havioral Neuroscience, 101, 587-590.

Rothblat, L. A., \& Kromer, L. F. (1991). Object recognition memory in the rat: The role of the hippocampus. Behavioural Brain Research, 42, 25-32.

Santucci, A. C., \& Treichler, F. R. (1990). Concurrent objectdiscrimination learning in rats. Animal Learning \& Behavior, 18, 295-301.

SQUIRE, L. R. (1992). Memory and the hippocampus: A synthesis from findings with rats, monkeys, and humans. Psychological Review, 99, 195-231.

Souire, L. R., Zola-Morgan, S., \& Chen, K. S. (1988). Human amnesia and animal model of amnesia: Performance of amnesic patients on tests designed for the monkey. Behavioral Neuroscience, 102, 210-221.

UNGERLEIDER, L. G., \& MishKin, M. (1982). Two cortical visual systems. In D. J. Ingle, M. A. Goodale, \& R. J. Mansfield (Eds.), The analysis of visual behavior (pp. 549-586). Cambridge, MA: MIT Press.

Wible, C. G., Shiber, J. R., \& Olton, D. S. (1992). Hippocampus, fimbria-fornix, amygdala, and memory: Object discrimination in rats. Behavioral Neuroscience, 106, 751-761.

Wood, E. R., Mumby, D. G., Pinel, J. P. J., \& Phillips, A. G. (1993). Impaired object recognition memory in rats following ischemiainduced damage to the hippocampus. Behavioral Neuroscience, 107, 51-62.

ZILLES, K. (1985). The cortex of the rat: A stereotaxic atlas. Berlin: Springer-Verlag.

Zola-Morgan, S., \& SQuire, L. R. (1985). Medial temporal lesions in monkeys impair memory on a variety of tasks sensitive to human amnesia. Behavioral Neuroscience, 99, 22-34.

Zola-Morgan, S., Squire, L. R., \& Amaral, D. G. (1989a). Lesions of the amygdala that spare adjacent cortical regions do not impair memory or exacerbate the impairment following lesions of the hippocampal formation. Journal of Neuroscience, 9, 1922-1936.

Zola-Morgan, S., Squire, L. R., \& Amaral, D. G. (1989b). Lesions of the hippocampal formation but not lesions of the fornix or the mammillary nuclei produce long-lasting memory impairment in monkeys. Journal of Neuroscience, 9, 898-913.

(Manuscript received September 9, 1993; revision accepted for publication June 29,1994 .) 